\title{
Estados Físicos e Condições Iniciais
}

\author{
Mario Cezar Bertin ${ }^{1}$ \\ ${ }^{1}$ Affiliation not available
}

November 12, 2020

\begin{abstract}
Nesta seção, introduziremos o conceito de estados físicos de um sistema em mecânica clássica.
\end{abstract}

\section{Introdução}

A mecânica clássica é considerada, ao mesmo tempo e por boas razões, um protótipo e uma teoria efetiva da física quântica. É uma questão aberta a forma como a mecânica quântica pode ser mapeada em seu análogo clássico de forma geral, mas em muitos sistemas físicos específicos há uma correspondência entre a descrição clássica e quântica. Contudo, como a mecânica clássica surgiu muito antes da mecânica quântica, muitos conceitos permanecem descorrelacionados entre ambas as visões de mundo, não por haver discordâncias fundamentais entre os pontos de vista, mas por tradicionalismo por parte da visão clássica. Outros conceitos foram modificados por necessidade empírica ou epistemológica.

Um dos conceitos que carregamos ainda hoje e que pode ser reformulado para ser análogo ao mesmo conceito em mecânica quântica, sem perder suas características ontológicas, é o conceito de estado físico. Na verdade, o conceito fundamental ainda é compartilhado por ambas as teorias.

Um estado físico de um sistema é determinado por um conjunto específico de características capaz de descrevê-lo completamente.

De forma mais precisa, um estado físico é um conjunto de medidas referentes a um conjunto de observáveis que possuem uma característica de completeza. A completeza de um conjunto de observáveis, por outro lado, é uma propriedade algébrica definida em teorias de grupos, assunto com o qual não nos preocuparemos aqui. Desde que um critério de completeza seja definido, o conjunto de observáveis que definem os estados físicos pode ser finito ou infinito. Existem sistemas clássicos cujo conjunto de observáveis definidores dos estados físicos é infinito. Contudo, os sistemas com os quais nos preocuparemos possuem sempre conjuntos finitos desses observáveis.

Tradicionalmente, o estado físico em mecânica clássica é definido por dois observáveis: a posição e a velocidade em determinado instante de tempo. Portanto, cada ponto da curva solução das equações de movimento de uma partícula, em conjunto com sua velocidade, determinam o estado da partícula. Assim, o conjunto

$$
E(t) \equiv\left\{x_{1}(t), x_{2}(t), x_{3}(t), \dot{x}_{1}(t), \dot{x}_{2}(t), \dot{x}_{3}(t)\right\}
$$

determina o estado de uma partícula com trajetória $\gamma: \mathbf{x}=\mathbf{x}(t) \subset \mathbb{R}^{3}$ no instante de tempo $t$. Veremos no formalismo Lagrangiano que a coleção de todos os estados definidos dessa forma forma um espaço vetorial, denominado espaço de estados. 
Aqui, vamos definir observáveis clássicos de outra forma. Contudo, ambas as definições são plenamente compatíveis. A diferença estará na maior proximidade entre a contrução que empregaremos abaixo e aquela que é definida na mecânica quântica.

\section{O conceito de estado físico a partir do oscilador harmônico}

Considere o oscilador harmônico simples unidimensional. Ele é descrito como uma partícula no potencial $V=$ $m \omega^{2} x^{2} / 2$. Assim, utilizando-se da relação $\dot{p}=-d V / d x$, temos a equação de movimento

$$
\ddot{x}+\omega^{2} x=0,
$$

que descreve o movimento do oscilador. Suas soluções determinam todas as possíveis curvas percorridas pela partícula em $\mathbb{R}$, ou seja, todas as possíveis formas pelas quais a dinâmica do sistema se realiza. A forma mais geral para a função horária solução da equação (??) é dada como uma combinação linear:

$$
x=a e^{i \omega\left(t-t_{0}\right)}+b e^{-i \omega\left(t-t_{0}\right)},
$$

em que

$$
e^{ \pm i \omega\left(t-t_{0}\right)}
$$

são soluções linearmente independentes de (??), como vimos no vídeo abaixo:

Rich media available at https://youtu.be/k8tQ712Ay1E

Como (??) é uma equação diferencial linear (EDO) de segunda ordem, admite duas soluções de base e a superposição de soluções como solução geral. A variável independente é $t$, mas a solução depende também de mais três parâmetros $a, b$ e $t_{0}$. O parâmetro $t_{0}$ é livre, pois é fixado pelo instante em que o observador liga seu relógio. Contudo, os demais parâmetros definem soluções particulares para o oscilador. Cada cojunto de valores $(a, b)$ distinto determina uma curva diferente para o oscilador. Dizemos, assim, que um conjunto específico $(a, b)$ define um estado físico acessível do sistema.

Pela teoria das EDOs lineares, vemos que a fixação dos parâmetros $(a, b)$ pode ser efetuada com a determinação de duas condições iniciais. Por exemplo, vamos supor que o oscilador seja um sistema massa-mola e que a posição inicial do oscilador seja determinada pelo experimentador estendendo-se a mola para uma amplitude $A$. Ele liga o cronômetro em $t=t_{0}$ enquanto libera a massa para se mover livremente. Neste caso, a posição inicial é dada por $x\left(t_{0}\right)=A$, o que resulta na condição

$$
a+b=A .
$$

Por outro lado, temos

$$
\dot{x}=i \omega a e^{i \omega\left(t-t_{0}\right)}-i \omega b e^{-i \omega\left(t-t_{0}\right)},
$$

que é a velocidade geral da partícula. Ao mesmo tempo em que o sistema inicia o movimento, sua velocidade é nula. Assim, a velocidade inicial promove a condição

$$
\dot{x}_{0}=\dot{x}\left(t=t_{0}\right)=0,
$$

o que implica em

$$
a-b=0
$$

Portanto, $b=a=A / 2$ e, assim, a solução passa a ser

$$
x=\frac{1}{2} A\left[e^{i \omega\left(t-t_{0}\right)}+e^{-i \omega\left(t-t_{0}\right)}\right]=A \cos \left[\omega\left(t-t_{0}\right)\right] .
$$


Assim, o estado do sistema é determinado pelo conjunto $(a, b)=(A / 2, A / 2)$, sendo definido por uma única constante $A$, a amplitude do movimento. Portanto, para cada valor da amplitude, temos definida uma solução distinta para o oscilador harmônico simples. Neste caso, o estado do oscilador é definido apenas pelo valor da amplitude.

Devemos nos lembrar, contudo, que a amplitude tem relação direta com a energia mecânica do sistema,

$$
A=\sqrt{\frac{2 E}{m \omega^{2}}}
$$

portanto, o estado físico do oscilador é determinado pela energia $E$. Isto pode ser visto também na solução encontrada em (Bertin, 2020).

Como a equação de movimento é de segunda ordem, são sempre necessárias duas condições para definir uma solução única, ou seja, um estado físico do sistema. Vamos voltar à solução

$$
x=a e^{i \omega\left(t-t_{0}\right)}+b e^{-i \omega\left(t-t_{0}\right)}
$$

do OHS como exemplo. Em vez de definir uma condição inicial, vamos supor que conhecemos o valor da posição em um instante de tempo

$$
t^{\prime}: t=t^{\prime}+t_{0}
$$

Neste caso,

$$
x^{\prime} \equiv x\left(t^{\prime}+t_{0}\right)=a e^{i \omega t^{\prime}}+b e^{-i \omega t^{\prime}}
$$

e

$$
\dot{x}^{\prime} \equiv \dot{x}\left(t^{\prime}+t_{0}\right)=i \omega a e^{i \omega t^{\prime}}-i \omega b e^{-i \omega t^{\prime}}
$$

o que resulta em

$$
a=\frac{1}{2}\left(x^{\prime}-\frac{i}{\omega} \dot{x}^{\prime}\right) e^{-i \omega t^{\prime}}, \quad b=\frac{1}{2}\left(x^{\prime}+\frac{i}{\omega} \dot{x}^{\prime}\right) e^{i \omega t^{\prime}}
$$

Esta é a forma geral dos coeficientes que determinam o estado físico do OHS, dependendo das condições iniciais $x=x^{\prime}$ e $\dot{x}=\dot{x}^{\prime}$. Esta análise nos permite definir as funções

$$
a(t) \equiv \frac{1}{2}\left(x-\frac{i}{\omega} \dot{x}\right) e^{-i \omega\left(t-t_{0}\right)},
$$

e

$$
a^{*}(t) \equiv b(t)=\frac{1}{2}\left(x+\frac{i}{\omega} \dot{x}\right) e^{i \omega\left(t-t_{0}\right)},
$$

que aparecem muito no problema quântico relacionado ao oscilador harmônico simples. Em mecânica quântica, essas funções são denominadas operadores de criação e destruição. Note que a denominação $a^{*}$ indica conjugação complexa, em que a base imaginária $i$ é trocada por $-i$ a partir da expressão de $a$.

Ainda sobre as funções $a$ e $a^{*}$, podemos mostrar que são, na verdade, constantes de movimento. Basta calcular

e

$$
\frac{d a}{d t}=-\frac{1}{2} \frac{i}{\omega}\left(\ddot{x}+\omega^{2} x\right) e^{-i \omega\left(t-t_{0}\right)}=0,
$$

$$
\frac{d a^{*}}{d t}=\frac{1}{2} \frac{i}{\omega}\left(\ddot{x}+\omega^{2} x\right) e^{i \omega\left(t-t_{0}\right)}=0,
$$

em que usamos as equações de movimento (??). Portanto, no caso de condições iniciais mais gerais, o estado físico do OHS é definido pelo par de constantes de movimento $\left(a, a^{*}\right)$, que desta vez são números complexos. 
Assim, como no caso mais restrito em que a condição inicial é $x_{0}=A$, no caso mais geral temos uma relação entre as funções $\left(a, a^{*}\right)$ e a energia mecânica. Note que

$$
a^{*} a=\frac{1}{4 \omega^{2}}\left(\omega^{2} x^{2}+\dot{x}^{2}\right)=\frac{E}{2 m \omega^{2}},
$$

ou seja,

$$
E=2 m \omega^{2} a^{*} a .
$$

É fácil mostrar que, no caso particular em que $x_{0}=A$ e $\dot{x}_{0}=0, a=a^{*}=A / 2$ e $a^{*} a=A / 4$.

Assim, novamente, apenas a energia é necessária para determinar o estado de movimento, visto que o oscilador em si determina as relações entre as condições iniciais e as constantes de movimento do sistema. Esta é uma relação que acompanhará a resolução de todos os problemas mecânicos integráveis, ou seja, que possuem soluções analíticas. Assim, os estados físicos de um sistema deverão ser definidos por um conjunto de observáveis que são constantes de movimento do sistema. Veremos melhor como isso é concretizado.

Como já destacamos, historicamente, o conceito de estado físico na mecânica clássica não é definido a partir dos observáveis invariantes. Para o conceito tradicionalmente aceito, inclusive em livros texto atuais, atribuímos um estado físico para cada valor de posição e momento. Assim, cada ponto da trajetória do oscilador, para o qual uma dupla $(x, \dot{x})$ é estabelecida, é um estado físico na concepção clássica. Sob este ponto de vista, cada ponto da trajetória é um estado físico. Sob o ponto de vista que abordamos aqui, cada valor de $E$ define um estado físico. Este último ponto de vista é na mecânica quântica e na teoria quântica de campos, portanto, possui uma conexão mais profunda com a física moderna.

\section{Os estados físicos do oscilador isotrópico}

\section{Rich media available at https://youtu.be/ibR8EySfRKc}

No caso do oscilador isotrópico tridimensional, a definição de estados físicos envolve também seus invariantes fundamentais: A energia e o momento angular. Lembremos que, em coordenadas esférico-polares, a energia do oscilado isotrópico é dada por

$$
E=\frac{1}{2} m \dot{r}^{2}+\frac{\ell^{2}}{2 m r^{2}}+\frac{1}{2} m \omega^{2} r^{2}
$$

em que $\ell^{2}$ é o momento angular ao quadrado do sistema,

$$
\ell^{2}=m^{2} r^{4}\left(\dot{\theta}^{2}+\dot{\phi}^{2} \sin ^{2} \theta\right)
$$

Vimos que, se o sistema de coordenadas for escolhido de modo que o eixo $\mathbf{e}_{3}$ coincida com a direção do momento angular $\mathbf{L}$, ou seja, se

$$
\mathbf{L}=L_{3} \mathbf{e}_{3}=m r^{2} \dot{\phi} \sin ^{2} \theta \mathbf{e}_{3},
$$

temos que

$$
\dot{\theta}=0 \quad \text { e } \quad \theta=\frac{\pi}{2},
$$

portanto,

$$
\ell=m r^{2} \dot{\phi}
$$

Ambos os valores $E$ e $\ell$ são invariantes dinâmicos, contudo, a energia possui espectro no intervalo $E \in[\omega \ell, \infty)$, enquanto $\ell \in(-\infty, \infty)$. Contudo, na equação (??) vemos que cada valor de energia depende de $\ell^{2} \in[0, \infty)$, portanto, um valor específico de energia pode ter infinitas soluções de momentos angulares distintos. Apenas com a definição de ambos os invariantes uma solução única pode ser definida. Neste caso, os observáveis que definem os estados físicos do sistema são a energia mecânica e o módulo do momento angular. 
Relacionar estes invariantes aos dados iniciais do oscilador não é tão simples como no caso unidimensional. Contudo, os estados definidos por $E$ e $\ell$ também são relacionados a condições iniciais do problema e, no caso mais geral, a funções análogas às funções de criação e destruição $a$ e $a^{*}$. Contudo, este problema foge ao escopo deste curso.

No caso mais geral, em que o eixo $\mathbf{e}_{3}$ não coincide com a direção do momento angular $\mathbf{L}$, ou seja, $L_{1} \neq 0$ e $L_{2} \neq 0$, esses dois observáveis não serão suficientes para definir completamente o estado do oscilador. Isto ocorre porque o movimento em $\theta$ não é mais fixado. Neste caso, haverá mais um invariante dinâmico fundamental. Vamos voltar ao problema do oscilador isotrópico tridimensional no formalismo lagrangiano. Então, os três invariantes serão mais facilmente obtidos, assim como suas devidas interpretações.

\section{References}

Mecânica Clássica - Quadraturas. (2020). https://doi.org/10.22541/au.160133732.21279283 